\title{
Residents' Perception of Skill Decay during Dedicated Research Time
}

\author{
Anne-Lise D. D'Angelo, MD ${ }^{1}$, Rebecca. D. Ray, $\mathrm{PhD}^{1}$, Caitlin G. Jenewein, $\mathrm{BS}^{1}$, Grace F. Jones ${ }^{1}$, \\ Carla. M. Pugh, MD, $\mathrm{PhD}^{1}$ \\ ${ }^{1}$ University of Wisconsin, School of Medicine and Public Health, Department of Surgery
}

\author{
Corresponding Author: \\ Anne-Lise D'Angelo, MD \\ Department of Surgery \\ University of Wisconsin Hospital and Clinics \\ 600 Highland Ave. \\ K6/135 CSC \\ Madison, WI 53792 \\ (608) 262-0466 \\ Ad'angelo@uwhealth.org
}

\section{Subject Category: Education}

\section{Author Contributions:}

Anne-Lise D'Angelo contributed to study conception and design; data collection, analysis and interpretation; writing the manuscript and critical revision of the manuscript.

Rebecca Ray contributed to $\backslash$ study conception and design; data collection, analysis and interpretation; writing the manuscript and critical revision of the manuscript.

Caitlin Jenewein contributed to study data collection, writing the manuscript and critical revision of the manuscript.

Grace Jones contributed to study data collection, writing the manuscript and critical revision of the manuscript.

Carla Pugh contributed to study conception and design; data collection, analysis and interpretation; writing the manuscript and critical revision of the manuscript 


\begin{abstract}
Introduction

Surgery residents may take years away from clinical responsibilities for dedicated research time. As part of a longitudinal project, the study aim was to investigate residents' perceptions of clinical skill reduction during dedicated research time. Our hypothesis was that residents would perceive a greater potential reduction in skill during research time for procedures they were less confident in performing.

Materials and methods

Surgical residents engaged in dedicated research training at multiple training programs participated in four simulated procedures: urinary catheterization, subclavian central line, bowel anastomosis and laparoscopic ventral hernia $(\mathrm{LVH})$ repair. Using pre and post-procedure surveys, participants rated procedures for confidence and difficulty. Residents also indicated the perceived level of skills reduction for the four procedures as a result of time in the laboratory.

Results

Thirty-eight residents (55\% female) completed the four clinical simulators. Participants had between 0-36 months in a laboratory $(\mathrm{M}=9.29$ months, $\mathrm{SD}=9.38)$. Pre-procedure surveys noted lower confidence and higher perceived difficulty for performing the LVH repair followed by bowel anastomosis, central line insertion, and urinary catheterization ( $\mathrm{p}<.05)$. Residents perceived the greatest reduction in bowel anastomosis and LVH repair skills compared to urinary catheterization and subclavian central line insertion $(\mathrm{p}<.001)$. Post-procedure surveys showed significant effects of the simulation scenarios on resident perception for urinary catheterization $(\mathrm{p}<.05)$ and LVH repair $(\mathrm{p}<.05)$.

Conclusion

Residents in this study expected greater skills decay for the procedures they had lower confidence performing and greater perceived difficulty. In addition, carefully adapted simulation scenarios had a significant effect on resident perception and may provide a mechanism for maintaining skills and keeping confidence grounded in experience.
\end{abstract}

Key words: Skill decay, Education, Resident, Simulation, Confidence, Self-efficacy 


\section{Introduction}

Concerns over resident readiness for operative independence have contributed to national discussions on the general surgery training curriculum [1-6]. Restructuring of the training curriculum [7], including changing the length of training [8], timing of specialization [8] and adding transition to care fellowships, is currently being debated [5-6]. Interestingly, there has been less discussion regarding the impact dedicated research time during residency has on surgical knowledge and skill acquisition. Residents that pursue research experiences in the middle of residency interrupt their clinical skills training prior to reaching mastery in certain skills. This may leave them vulnerable for skills decay and contribute to a lack of competency at the end of residency training.

General surgery training is unique in that many programs offer residents the opportunity to perform one to three years of dedicated research time during the middle of residency training [9]. Almost $40 \%$ of residents training at Accreditation Council for Graduate Medical Education (ACGME) and National Resident Matching Program (NRMP) affiliated programs spend at least one year engaged in research [10]. The vast majority of residents start their dedicated research time after two or three years of clinical training [10]. Although residents engaged in dedicated research training are significantly more likely to pursue fellowship training and less likely to hold private practice positions, little is known about how this research time affects operative performance [10-11].

Residents who pursue research experience may return to clinical practice with less surgical knowledge and skill than their peers who do not take time away for research. Factors known to contribute to skills decay include time away from task performance; level of knowledge mastery prior to time away and task characteristics [12-13]. There is a lack of research on skills decay during surgical research fellowships. Moreover, it is not known how skills decay during research fellowships impacts resident competency at 
the end of their training. Evaluating the potential reduction in surgical skill and knowledge during dedicated research time is critical to ensuring competent, independent performance at the end of training.

The aim of this study was to investigate residents' perceptions of clinical skill reduction during dedicated research time. We sought to evaluate the relationship between residents' perceived skill reduction, procedural confidence and perceived task difficulty before and after performing simulation-based procedures. Our hypothesis was that residents would perceive a greater potential reduction in skill during research time for procedures they were less confident in performing. Our hypothesis is in line with other research noting that previously mastered skills are less prone to skills decay [12].

\section{Methods}

\section{Setting and participants}

Study participants $(\mathrm{N}=38)$ were residents engaged in dedicated laboratory time from multiple general surgery training programs enrolled in a longitudinal simulation-based assessment study. This manuscript evaluates data from the first data collection period of a longitudinal simulation based assessment study. Residents that were currently in their clinical training were excluded from this study. Data collection occurred at five sites in three Midwestern cities: Madison, WI, Chicago, IL, and Rochester, MN.

This study was approved by the University of Wisconsin Institutional Review Board and written informed consent was obtained from all participants.

Surveys

General survey 
Prior to performing the simulated procedures, residents completed a general survey designed to collect information on demographics, years of general surgery training, years of dedicated laboratory work and current call schedule. This survey also used a 5 point Likert scale to assess perceived reduction in global clinical and surgical skills and procedure specific (urinary catheterization, subclavian central line insertion, bowel anastomosis, and LVH repair) performance during dedicated laboratory time.

Procedure related surveys

Following the general survey, residents completed a pre-procedure survey designed to assess confidence in and perceived difficulty in performing specific procedures steps and the entire surgical task. Survey items for each procedure are as follows: urinary catheterization (identify anatomy; problem solve difficulties with insertion; successfully perform entire procedure); subclavian central line insertion (identify landmarks; cannulate the subclavian vein; successfully perform entire procedure), bowel anastomosis (select correct suture; select correct stitch; successfully perform entire procedure), and LVH repair (plan port placement; completely visualize the hernia defect; successfully perform entire procedure $)$. Confidence and perceived difficulty was assessed using a 5-point Likert scale $(1=$ Not confident; 5 = Extremely confident; and 1 = Not difficult; $5=$ Extremely difficult respectively). After completing each simulated procedure, residents completed a post-procedure survey designed to re-assess confidence and perceived difficulty of performing the surgical tasks. The same questions and 5-point Likert scales were used in the pre-procedure survey and post-procedure survey.

\section{Simulated procedures}

After completing the general survey and pre-procedure survey, participants completed the four simulated clinical procedures: urinary catheterization, subclavian central line insertion, bowel anastomosis and 
laparoscopic ventral hernia (LVH) repair. These procedures were purposefully selected and designed to provide opportunities for both decision making and technical skill performance. Participants had 15 minutes to complete each simulated task with additional transition time between stations. This amount of time was selected to balance data collection requirements with participant fatigue. Simulator development was based on prior cognitive task analysis [14-16] and all stations were reviewed by experts prior to data collection. The focus of each station was predetermined to allow for participants to engage in major decisions and technical hurdles. The procedures were randomized with a Latin square. Prior to starting each station, researchers read an introductory narrative providing information on the simulator and the station task. Next, participants were read a patient scenario prior to starting the procedure.

\section{Urinary catheterization}

This station was designed to represent four different patient scenarios (two female, two male) requiring urinary catheterization. Each participant was presented with three of the four possible patient scenarios in a randomized fashion. The patient scenarios included a 27 year old female trauma patient; 45 year old female operative patient; 74 year old male with rectal cancer; and 67 year old post-operative male with urinary retention. Patient scenarios were randomized by data collection site. The task in each scenario was to place a urinary catheter. Participants were informed that the perineum had already been prepped in the usual sterile fashion. Five urinary catheters (16 French (Fr) Foley, 16 Fr Coude, 16 Fr 3-way Foley, 12 Fr Foley and 10 Fr Foley) were available for participants to use during this station along with lubricant and sterile water. Participants also had the option to place a urology consult. We theorized the urinary catheterization station would be easier from a technical skills aspect. This led us to include the choice of catheter in the simulation, and a wide variation in clinical presentations.

Subclavian central line insertion 
This station was designed to represent a hypotensive, tachycardic and febrile patient in need of central venous access. The task was to place a subclavian central line. The simulator (CentralLineMan System, Simulab Corp, Seattle, WA) consisted of a realistic anatomical representation of a right upper adult torso with internal and external jugular and subclavian veins; carotid and subclavian arteries and venous and arterial "blood". The simulator was placed in Trendelenburg position. Participants were informed that the patient had been prepped and draped in the usual sterile fashion and the skin had been injected with local anesthetic. A complete central line insertion kit with all the necessary equipment to perform a subclavian central line insertion, except an ultrasound machine, was provided. We theorized that residents would have varying levels of skill in performing this procedure. This led us to make a decision to allow the entire natural, built in complexity of central line placement to stand 'as is'. We expected a wide variety of performance and completion rates in the allotted time. Safety in needle angle and maintenance of sterility are two examples of important decisions and possible errors.

Bowel anastomosis

This station was designed to represent a trauma patient in the operating room with an abdominal gunshot wound. The task was to inspect and repair the injured bowel. The simulator consisted of bovine intestine arranged on a tray with accompanying artificial blood and a single bullet. Two full thickness injuries in close proximity were located at the anti-mesenteric border [17]. One injury was larger $(1 \mathrm{~cm} \times 1 \mathrm{~cm})$ with jagged edges and the other injury was smaller $(0.5 \mathrm{~cm} \times 0.5 \mathrm{~cm})$. A trained researcher considered to be at the level of a medical student acted as a surgical assistant during the simulated procedure. Except electrocautery and stapler devices, all necessary open surgical instruments along with a selection of suture (3-0 Vicryl® (Ethicon), Nylon, Silk, PDS® (polydioxanone, Ethicon), and Prolene $($ Ethicon)) were provided to complete the repair. Participants were required to decide how to repair the injury. We 
theorized that residents would have less experience independently completing this task. Choice of repair technique, suture selection, and stitch selection provided multiple decision points for participants to make.

Laparoscopic ventral hernia (LVH) repair

This station was designed to represent an intra-operative patient with a ventral hernia. The majority of the exposure had already taken place. The task was to perform a portion of the LVH repair by securing the mesh with the use of transfacial sutures and a tacker. The simulator is composed of a base covered with a simulated abdominal wall including a $10 \mathrm{~cm} \times 10 \mathrm{~cm}$ ventral hernia [14-16]. Participants were informed that abdominal access had been achieved, the abdomen was insufflated, ports had been placed and a piece of mesh inserted in the abdomen. The abdominal mesh was a $16 \times 16 \mathrm{~cm}$ piece with four anchoring sutures already in place. Two of the four transfacial sutures had already been brought up through the abdominal wall and secured with hemostats. Participants were instructed to bring up the remaining transfacial sutures using a suture passing device and place the first five securing tacks. A tray of all of the necessary open and laparoscopic instruments, except electrocautery, was available for use by the participants. A trained researcher considered to be at the level of a medical student acted as the operative assistant. We theorized that residents would have a wide range of actual experience as first assistant and operative surgeon at this step of the procedure. To assess port placement decisions, participants were required to diagram port location for laparoscopic hernia repairs on paper prior to starting the simulation. Proficient use of laparoscopy and coordination with the suture passer device would be required to achieve adequate hernia coverage and well placed mesh. These steps provided cognitive and technical complexity at this station. Ports were pre-placed to provide participants with the maximal amount of time to perform mesh securing and tacking.

\section{Data analysis}


Demographic variables were averaged. Participant responses to pre-procedure and post-procedure survey confidence and perceived difficulty ratings were averaged and means compared with repeated measures analysis of variance (ANOVA) and paired t-tests. Pearson correlations were used to test associations between perceived skill reduction, total months spent in the laboratory, and pre and post-procedural confidence and difficulty ratings.

\section{Results}

\section{Demographics}

Thirty eight surgery residents, engaged in dedicated laboratory work (55\% female), participated in the study. Residents had already completed $1(n=1) ; 2(n=25)$; or $3(n=11)$ clinical years at the time of participation in the study. One participant did not indicate number of clinical years completed. Overall, residents had completed an average of 9.29 months $(\mathrm{SD}=9.38)$ in the laboratory and planned to spend a mean of 16.32 additional months $(\mathrm{SD}=8.40)$ doing research at the time of study participation.

The majority of residents (66\%) had on-call responsibilities. Median on-call responsibilities was 1-2 shifts per month. A minority of residents (37\%) were engaged in moonlighting work during their laboratory time. Those that performed moonlighting work had a median number of 3-4 shifts per month. Figure 1 depicts the percentage of residents that performed bedside or surgical procedures during on-call or moonlighting work.

\section{Perceived skill reduction}

Residents' perceived reduction in both global clinical and procedure specific surgical skills are displayed in Figure 2. Amongst procedure specific skills, residents perceived a greater reduction in bowel 
anastomosis $(\mathrm{M}=3.0, \mathrm{SD}=0.96)$ and $\mathrm{LVH}$ repair $(\mathrm{M}=2.84, \mathrm{SD}=1.03)$ skills compared to subclavian central line insertion $(\mathrm{M}=2.13, \mathrm{SD}=0.81)$ and urinary catheterization $(\mathrm{M}=1.50, \mathrm{SD}=0.51)(\mathrm{F}(1,37)=36.59, \mathrm{p}<$ .001) (Figure 2). The biggest perceived reduction in global clinical skills was in technical surgical skills $(\mathrm{M}=3.19, \mathrm{SD} 0.95)$ and knowledge of procedure steps $(\mathrm{M}=3.00, \mathrm{SD}=0.76, \mathrm{~F}(6,210)=34.44, \mathrm{p}<.001$, all pair wise comparisons $\mathrm{p}<.01$ ). The months residents had already spent in the lab correlated with greater perceived reduction in technical surgical skills $(\mathrm{r}(37)=.40, \mathrm{p}=.007)$ and knowledge of procedure steps $(\mathrm{r}(37)=.36, \mathrm{p}=.015)$.

Pre and post-procedure confidence and difficulty ratings

Participants reported the most pre-procedure confidence for successfully performing the urinary catheterization procedure ( $\mathrm{M}=4.24$ "Very Confident", $\mathrm{SD}=0.71)$, followed by subclavian central line insertion ( $\mathrm{M}=3.37$ "Moderately Confident", $\mathrm{SD}=0.91)$, bowel anastomosis ( $\mathrm{M}=2.40$ "Somewhat Confident", $\mathrm{SD}=1.08)$ and $\mathrm{LVH}$ repair $(\mathrm{M}=2.13$ "Somewhat Confident", $\mathrm{SD}=1.02)(\mathrm{F}(3,111)=37.22 \mathrm{p}$ $<.001$, all pair wise comparisons $\mathrm{p}<.05$, Figure 3 ). Similarly, participants predicted that the urinary catheterization task would be the least difficult ( $M=1.22$ "Not Difficult", $\mathrm{SD}=0.42)$, followed by the subclavian central line insertion $(\mathrm{M}=2.11$ "Somewhat Difficult", $\mathrm{SD}=0.74)$, bowel anastomosis $(\mathrm{M}=2.97$ "Moderately Difficult, $\mathrm{SD}=0.83)$ and $\mathrm{LVH}$ repair (M=3.42 "Moderately Difficult", $\mathrm{SD}=1.08, \mathrm{~F}$ $(3,102)=39.59, \mathrm{p}<.001$, all pair wise comparisons $\mathrm{p}<.005$, Figure 4$)$. These findings are consistent with our hypothesis regarding procedure experience.

Comparisons of pre- and post-procedure confidence are detailed in Figures 3a-d. After completing the simulated surgical tasks, participants reported significantly decreased confidence performing the entire urinary catheterization task $(\mathrm{M}=3.16, \mathrm{SD}=1.05, \mathrm{t}(37)=6.05, \mathrm{p}<.001)$, as well as, specific procedure steps (identifying the relevant anatomy $(\mathrm{M}=3.92, \mathrm{SD}=0.82, \mathrm{t}(37)=3.47, \mathrm{p}=.001)$ and problem solving difficulties with urinary catheter insertion $(\mathrm{M}=3.24, \mathrm{SD}=0.88, \mathrm{t}(37)=5.28, \mathrm{p}<.001))$. Participant 
confidence improved for completely visualizing the hernia defect $(\mathrm{M}=3.11, \mathrm{SD}=1.11, \mathrm{t}(37)=-2.66$, $\mathrm{p}=.012$ ) on the LVH task. All other average confidence ratings remain basically unchanged for the other simulated procedures.

Comparisons of pre- and post-procedure difficulty are detailed in Figures 4a-d. Similar to the confidence ratings, participants rated the urinary catheter task (identifying the anatomy, problem solving difficulties with insertion of the catheter and the successful completion of the entire task) as more difficult than predicted (all p-values <.01). Regarding central line, identifying the relevant landmarks was also rated as more difficult than expected $(\mathrm{M}=1.87, \mathrm{SD}=0.78, \mathrm{t}(36)=-7.94, \mathrm{p}<.001)$. Conversely, after performing the simulation tasks, the LVH task (planning port placement, completely visualizing the hernia, and successfully performing the entire task) was rated as less difficult than previously predicted (all p-values <.05). Participants also rated selecting the correct suture for the bowel anastomosis as less difficult than expected $(\mathrm{M}=2.08, \mathrm{SD}=1.05, \mathrm{t}(36)=2.22, \mathrm{p}=.033)$.

Residents who reported engaging in surgical procedures during on call responsibilities, were more likely to have higher pre- and post-procedure confidence in the bowel anastomosis $(\operatorname{pre} \mathrm{r}(37)=.455, \mathrm{p}=.004$; post $\mathrm{r}(37)=.405, \mathrm{p}=.012$ ); higher pre- and post-procedure confidence in conducting the entire LVH (pre $\mathrm{r}(37)=.488, \mathrm{p}=.002$; post $\mathrm{r}(37)=.334, \mathrm{p}=.041)$. Engaging in surgical procedures also negatively correlated with difficulty ratings both pre and post procedure for the bowel anastomosis (Bowel pre r(37)=-.390, $\mathrm{p}=.017$, post $\mathrm{r}(37)=-.497, \mathrm{p}=.002)$ and LVH repair $(\mathrm{LVH}$ pre $\mathrm{r}(37)=-.398, \mathrm{p}=.016$, post $\mathrm{r}(37)=-.331$, $\mathrm{p}=.042$ ). There were no significant correlations between on call surgical or bedside procedures and confidence or difficult performing the subclavian central line or urinary catheterization. There were no relationships between whether or not someone engaged in either bedside or surgical moonlighting procedures and confidence or difficulty ratings. 
There were no significant correlations between months spent in the laboratory and perceived confidence and difficulty pre- and post-simulation or changes in confidence and difficulty ratings before and after performing the simulation tasks.

\section{Pre-procedure confidence and confidence change}

Confidence ratings prior to the simulation tasks correlated negatively with the amount of change in confidence in performing three of the four tasks (Table 1). Lower levels of confidence for the urinary catheterization, central line insertion and bowel anastomosis predicted increased confidence in performing the entire procedure after the simulation (all p-values <.05).

\section{Perceived skill reduction and resident confidence and procedural difficulty}

Residents perceived greater skills reduction for those procedures that they had lower confidence in

performing (Table 2). Specifically, predicted skill reduction in placing a subclavian central line negatively correlated with both pre and post-confidence ratings for performing the subclavian central line task (p $<.005)$. Perceived skill reduction placing a urinary catheter correlated negatively with pre-procedure confidence ratings only for the urinary catheter task $(\mathrm{p}<.005)$ and bowel anastomosis $(\mathrm{p}<.005)$. Predicted skill reduction performing a laparoscopic ventral hernia repair was unrelated to either pre- or post-simulation confidence ratings for performing the LVH task.

The more skill reduction participants predicted in performing a subclavian central line insertion, the more difficult they rated completing the task both before and after performing the task (all p-values <.05, Table 2). Skill reduction ratings for the bowel anastomosis correlated with pre-simulation task difficulty $(\mathrm{r}(37)=.34, \mathrm{p}=.038$, Table 2$)$, however, not with post-simulation task difficulty ratings. This can be interpreted to mean that the more bowel anastomosis skill reduction one predicted, the more difficult one 
perceived the entire task prior to the simulation. LVH skill reduction correlated with difficulty ratings of successfully performing the entire LVH task post-simulation $(\mathrm{r}(37)=0.32, \mathrm{p}<.05$, Table 2$)$.

\section{Discussion}

This study evaluated residents' perception of skill reduction during their time in the laboratory. Data were collected during the first time point of a longitudinal simulation-based skills assessment study.

Participants in this study were residents at multiple general surgery training programs who were engaged in dedicated research fellowships. We found residents had varying perceptions of skill reduction during dedicated research fellowships based on procedure type. Additionally, performance of a simulated procedure differentially influenced their ratings of confidence and task difficulty based on procedure type and task focus. Tasks were purposefully modified to allow for a variety of task planning decisions and technical complexities.

Prior to performing the simulated procedures, residents perceived the greatest skill reduction for bowel anastomosis followed by LVH repair, subclavian central line insertion and lastly urinary catheter insertion. The majority of residents in this study had completed two or three clinical years at the time of participation. Based on their level of training, these residents may not have reached mastery on the LVH repair or bowel anastomosis procedures. This is supported by our results demonstrating lower confidence and higher perceived difficulty for performing the LVH repair and bowel anastomosis than urinary catheterization or central line insertion. Prior work in skills decay has found that the amount of overlearning (or mastery) is a critical determinant of skill and knowledge retention $[12,18]$. Overlearning is training beyond that required for proficiency. Residents that have not had opportunities for overlearning on certain tasks such as the bowel anastomosis or LVH repair would be expected to have lower confidence and perceive higher risks of skills decay. Further work is needed to determine if their perception has any effect on performance. 
Residents who had on call responsibilities and engaged in surgical procedures reported higher preprocedure and post-procedure confidence for performing the bowel anastomosis and LVH repair. This indicates that exposure to surgical procedures during dedicated research time may allow for maintenance of self-efficacy and potentially maintenance of proficiency. There were no correlations between engagement in on call surgical procedures and urinary catheterization and subclavian line confidence or difficulty ratings. This may relate to the fact that residents were overall more confident in performing these procedures and expected less skill decay. Periodic exposure to surgical tasks decreases the retention interval and may support proficiency maintenance during time away from training $[12,19]$.

Residents also believed that they would have the greatest reduction in global technical surgical skill and knowledge of procedure steps. This perceived skill reduction was correlated with time already spent in the laboratory. Compared with clinical diagnostic, triage, documentation and team communication skills; residents may have less experience with technical surgical skills, and knowledge of procedure steps by the end of their second or third clinical year. This is consistent with the overlearning theory of skills decay. If residents have not had the opportunity for overlearning, global skills as well as procedure specific skills may be at risk for skills decay during dedicated research fellowships [12].

In the absence of any feedback on their performance, participants' pre- and post-procedure confidence and difficult ratings changed according to actual task complexity. For example, the urinary catheter procedure was purposefully made complex. Prior to performing the procedure, participants rated the urinary catheterization as having no to very little skills reduction along with high confidence and low difficulty ratings. However, as expected there were significant reductions in confidence and increases in difficulty ratings for all three items related to urinary catheter insertion (identifying the anatomy, problem solving difficulties with insertion of the catheter and the successful completion of the entire task). Conversely, the LVH task was shortened and made to focus on a small part of the procedure. Prior to 
performing the procedure, participants rated the LVH repair as having moderate skills reduction, somewhat to moderate confidence ratings and moderately to very difficult ratings. As expected, after performing the LVH simulated procedure, there were significant increases in confidence and decreases in difficulty ratings. This indicates that specific modifications of a performing a simulated task can influence ratings of procedure confidence and difficulty. Residents may become more confident on tasks that were easier than expected and become less confident on tasks that were more difficult than expected. Prior work has shown that during periods of inactivity, self-efficacy can be maintained even though skill performance declines [20]. Participation in periodic skills evaluations combined with simulation based training can be useful to maintain the relationship between self-efficacy and actual performance. Additionally, it is important to be cognizant of simulation scenario design for decision making and technical skills as it may impact residents' self-perceptions of skill even in the absence of feedback.

Study limitations largely relate to the simulated tasks and scenarios. Residents in this study performed four simulations that were chosen to represent a range of expected mastery for their skill level. However, these procedures are a small subset of possible clinical scenarios that a resident might see in surgical practice. As such, our results may not be generalizable across all procedure and expectations for mastery. Furthermore, the simulation exercises were designed to be conducted within a specific time frame. This may have influenced how residents' performed the task and possibly affected the difficulty of the procedure. However, we purposefully designed the simulations to contain opportunities for both decision making and technical performance in the amount of time provided. This study focuses on self-reported measures that may not correspond with objective performance. Residents' perception of their performance may be different than their actual performance. In addition, feedback was not provided prior to completion of the post-surveys. Lastly, we may not have found significant relationships between moonlighting surgical procedures and confidence or difficulty ratings given our small sample size which limits statistical power. 
This study showed that residents expect moderate skills decay on more difficult tasks (bowel anastomosis and LVH repair) and for technical surgical skills and knowledge of procedure steps. Despite this expectation for skills reduction during dedicated research fellowships, few programs have focused clinical skills curricula to reduce skills decay or remediate performance. Prior research has demonstrated that training to mastery has a protective effect for skills decay [21]. Those skills that residents have not trained to mastery may be more vulnerable to skills decay and require additional work for remediation.

Incorporation of these activities in resident training may inform training interventions that can be employed throughout dedicated research time. Our results show that simulation-based assessments and training affect resident perception of skill and may provide a mechanism for maintaining skills [18] and keeping confidence grounded in performance. 


\section{Acknowledgements}

We would like to thank Patrick Barlow, $\mathrm{PhD}$ for his contribution to survey design and data collection. Funding for this study came from the Department of Defense grant \#W81XWH-13-1-0080 entitled, "Psychomotor and Error Enabled Simulations: Modeling Vulnerable Skills in the Pre-Mastery Phase" and NIH grant \#1F32EB017084-01 entitled "Automated Performance Assessment System: A New Era in Surgical Skills Assessment”. 


\section{Disclosures:}

Funding for this study came from the Department of Defense grant \#W81XWH-13-1-0080 entitled, "Psychomotor and Error Enabled Simulations: Modeling Vulnerable Skills in the Pre-Mastery Phase" and NIH grant \#1F32EB017084-01 entitled "Automated Performance Assessment System: A New Era in Surgical Skills Assessment". 


\section{References}

[1] Coleman JJ, Esposito TJ, Rozycki GS, et al. Early subspecialization and perceived competence in surgical training: are residents ready? J Am Coll Surg, 2013;216(4):764-71.

[2] Nakayama DK, Taylor SM. SESC Practice Committee survey: surgical practice in the duty-hour restriction era. Am Surg, 2013;79(7):711-5.

[3] Bucholz EM, Sue GR, Yeo H, Roman, SA, Bell RH Jr, Sosa, JA. Our trainees' confidence: results from a national survey of 4136 US general surgery residents. Arch Surg, 2011;146(8):907-14.

[4] Mattar SG, Alseidi AA, Jones DB, Jeyarajah, DR, Swanstrom, LL, Aye, RW, et al. General surgery residency inadequately prepares trainees for fellowship: results of a survey of fellowship program directors. Ann Surg, 2013;258(3):440-9.

[5] Richardson, J.D. ACS transition to practice program offers residents additional opportunities to hone skills. The Bulletin of the American College of Surgeons. http://bulletin.facs.org/2013/09/acs-transitionto-practice-program-offers-residents-additional-opportunities-to-hone-skills/. Published 2013. Accessed March 6 ${ }^{\text {th }}, 2014$.

[6] Hoyt, B. D. Executive Director's report: Looking forward - February 2013. Bulletin of the American College of Surgeons 2013. http://bulletin.facs.org/2013/02/looking-forward-february-2013/. Published 2014. Accessed March 6 ${ }^{\text {th }}, 2014$.

[7] Lewis FR, Klingensmith ME. Issues in general surgery residency training-2012. Annals of Surgery 2012; 256: 553-55

[8] Klingensmith, ME, Lewis, FR. General surgery residency training issues. Advances in Surgery 2013;47:251-270

[9] Roberston, CM, Klingensmith, ME, Coopersmith, CM. Long-term outcomes of performing a postdoctoral research fellowship during general surgery residency. Annals of Surgery 2007;245(4):516523.

[10] Roberston, CM, Klingensmith, ME, Coopersmith, CM. Prevalence and cost of full-time research fellowships during general surgery residency. Annals of Surgery 2009;249(1):155-161.

[11] Ellis, MC, Dhungel, B, Weerasinghe, R, Vetto, JT, Deveney, K. Trends in research time, fellowship training, and practice patterns among general surgery graduates. Journal of Surgical Education 2011;68(4):309-312.

[12] Arthur Jr, W, Bennett Jr, W, Stanush, PL, McNelly, TL. Factors that influence skill decay and retention: A qualitative review and analysis. Human Performance 1998;11(1):57-101.

[13] Schendel, JD, Shields, JL, Katz, MS. Retention of motor skills: Review. JSAS Catalog of Selected Documents in Psychology 1980;10(21). (Ms. No. 2016)

[14] Pugh CM, DaRosa DA, Santacaterina S, Clark, RE. Faculty evaluation of simulation-based modules for assessment of intraoperative decision making. Surgery 2011;149(4):534-42. 
[15] Pugh C, Plachta S, Auyang E, Pryor, A, Hungness, E. Outcome measures for surgical simulators: is the focus on technical skills the best approach? Surgery 2010;147(5):646-54.

[16] Pugh CM, Santacaterina S, DaRosa DA, Clark RE. Intra-operative decision making: more than meets the eye. Journal of Biomedical Informatics 2011;44:486-496.

[17] D'Angelo, AD, Cohen, ER, Kwan, C, Laufer, S, Greenberg, C, Greenberg, J, et al.Use of decisionbased simulations to assess resident readiness for operative independence. Am J Surg 2015;209(1):132139.

[18] Baldwin, TT, Ford, JK. Transfer of training: A review and directions for future research. Personnel Psychology 1988; 41(1), 63-105.

[19] Stefanidis, D, Korndorffer, JR, Markley, S, Sierra, R, Scott, D. Proficiency maintenance: Impact on ongoing simulator training on laparoscopic skill retention. J Am Coll Surg 2005; 202(4), 599-603.

[20] Youngqiust, ST, Henderson, DP, Gausche-Hill, M, Goodrich, SM, Poore, PD, Lewis, RJ. Paramedic self-efficacy and skill retention in pediatric airway management. Acad Emerg Med 2008;15(12):1295-

1303.

[21] Hagman, JD, Rose, AM. Retention of military tasks: A review. Human Factors 1983;25(2):199-213. 


\section{Figure legends}

Figure 1. Proportion of residents engaged in procedures during on-call and moonlighting shifts.

Figure 2. Average predicted reduction in procedure specific and global clinical and surgical skills.

Figure 3. Average pre and post-procedure confidence for four procedures (central line, bowel anastomosis, urinary catheterization and laparoscopic ventral hernia (LVH) repair).

Figure 4. Average pre and post-procedure perceived difficulty for four procedures (central line, bowel anastomosis, urinary catheterization and laparoscopic ventral hernia (LVH) repair). 
Table 1. Correlations between pre-procedure confidence and the change in confidence before and after the simulated procedure.

\begin{tabular}{lll}
\hline Procedure & $\begin{array}{l}\text { Correlation with pre- } \\
\text { procedure confidence }\end{array}$ & p-value \\
\hline Urinary catheterization & & \\
$\quad$ Identify relevant anatomy & -.237 & .076 \\
$\quad$ Problem solve difficulties inserting catheter & -.368 & $.011^{*}$ \\
$\quad$ Successfully perform entire task & -.388 & $.008^{* *}$ \\
Subclavian central line & & \\
$\quad$ Identify appropriate landmarks & -.301 & $.033^{*}$ \\
Cannulate subclavian vein & -.230 & .083 \\
$\quad$ Successfully perform entire task & -.299 & $.034^{*}$ \\
Bowel anastomosis & & \\
$\quad$ Select correct suture & -.225 & .090 \\
$\quad$ Select correct stitch & -.305 & $.031^{*}$ \\
$\quad$ Successfully perform entire task & -.470 & $.001^{* * *}$ \\
Laparoscopic ventral hernia repair & & .085 \\
$\quad$ Plan proper port location & -.227 & .123 \\
Completely visualize hernia defect & -.193 & .203 \\
$\quad$ Successfully perform entire task & -.139 & \\
\hline
\end{tabular}


Table 2. Correlations of perceived skill reduction and pre and post-procedure confidence and difficulty ratings for four procedures.

\begin{tabular}{lcccc}
\hline & \multicolumn{2}{c}{ Confidence } & \multicolumn{2}{c}{ Difficulty } \\
\hline Perceived skill reduction & $\begin{array}{c}\text { Pre- } \\
\text { procedure }\end{array}$ & $\begin{array}{c}\text { Post- } \\
\text { procedure }\end{array}$ & $\begin{array}{c}\text { Pre- } \\
\text { procedure }\end{array}$ & $\begin{array}{c}\text { Post- } \\
\text { procedure }\end{array}$ \\
\hline Urinary catheterization & $-.560^{* * *}$ & -.051 & .117 & -.228 \\
Subclavian central line & $-.578^{* * *}$ & $-.582^{* * *}$ & $.346^{*}$ & $.368^{*}$ \\
Bowel anastomosis & $-.496^{* *}$ & -.275 & $.343^{*}$ & .134 \\
Laparoscopic ventral hernia repair & -.186 & -.065 & -.023 & $.321^{*}$ \\
\hline
\end{tabular}




\section{Figure 1}

㣂 Bedside procedures
NSurgical procedures
鯤 Both bedside and surgical procedures
No procedures while on call or moonlighting
- No call or moonlighting responsibilities




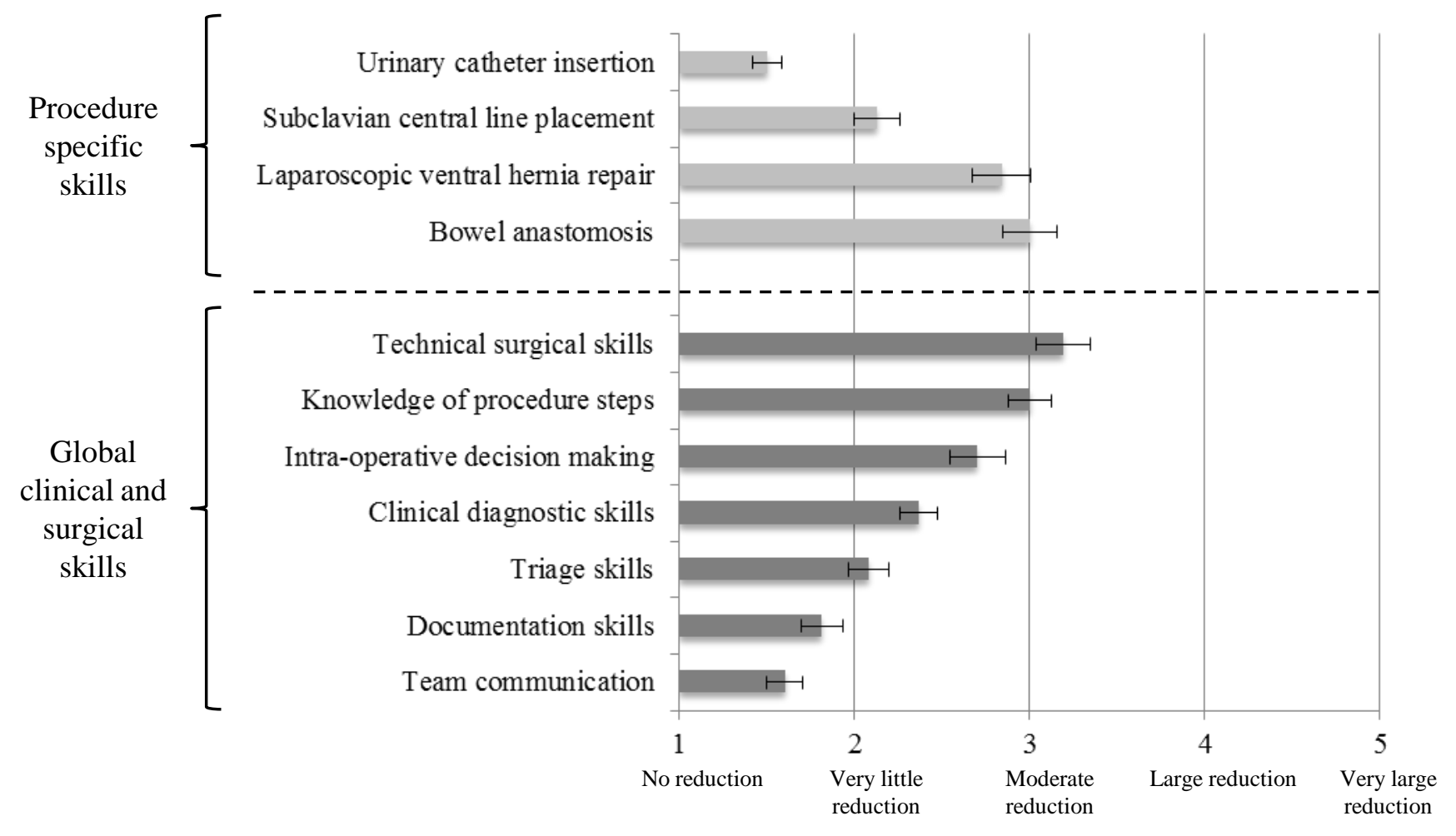




\section{a. Urinary catheterization}

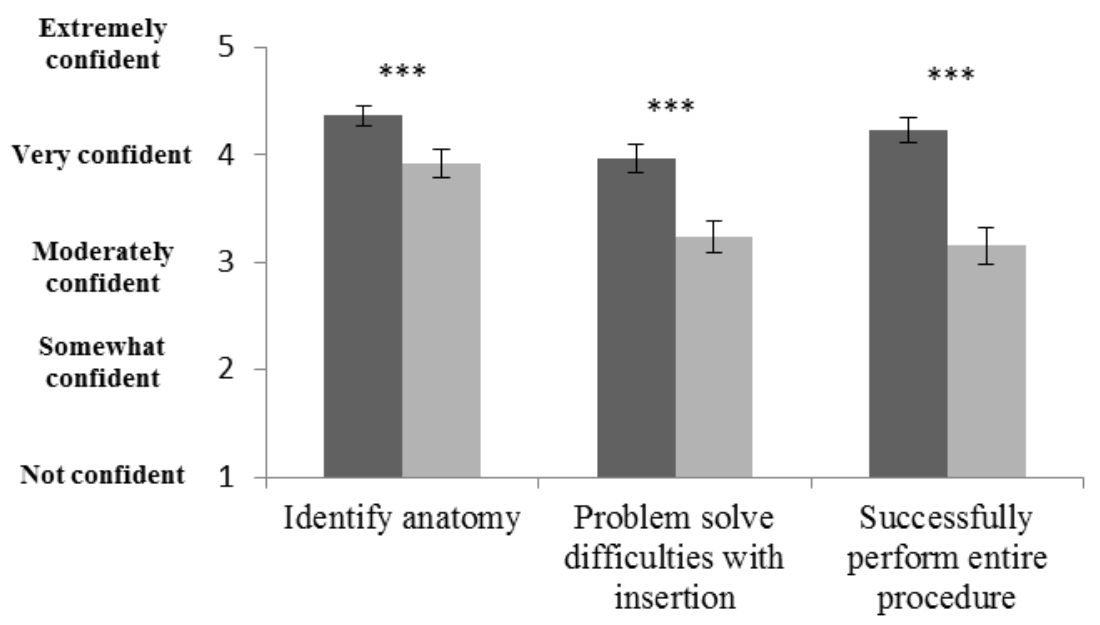

\section{c. Bowel anastomosis}



\section{b. Subclavian central line}

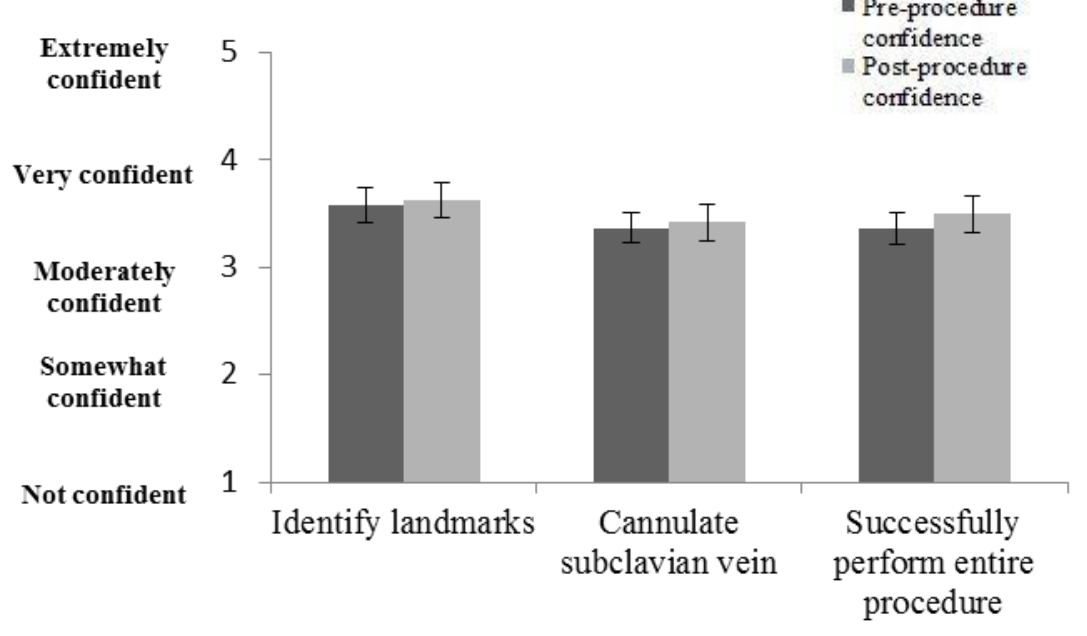

\section{d. LVH repair}



$$
\begin{aligned}
& * * \mathrm{p}<.01 \\
& * * * \mathrm{p}<.001
\end{aligned}
$$




\section{a. Urinary catheterization}



\section{c. Bowel anastomosis}

Extremely 5

difficult

Very

difficult

Moderately difficult

Somewhat difficult

Not difficult

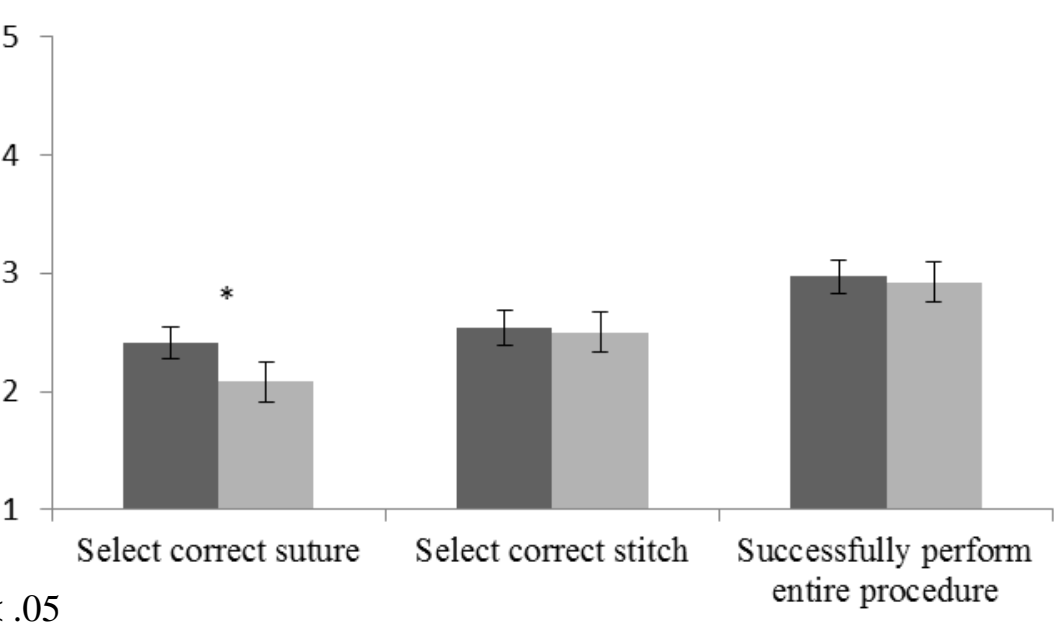

\section{b. Subclavian central line}

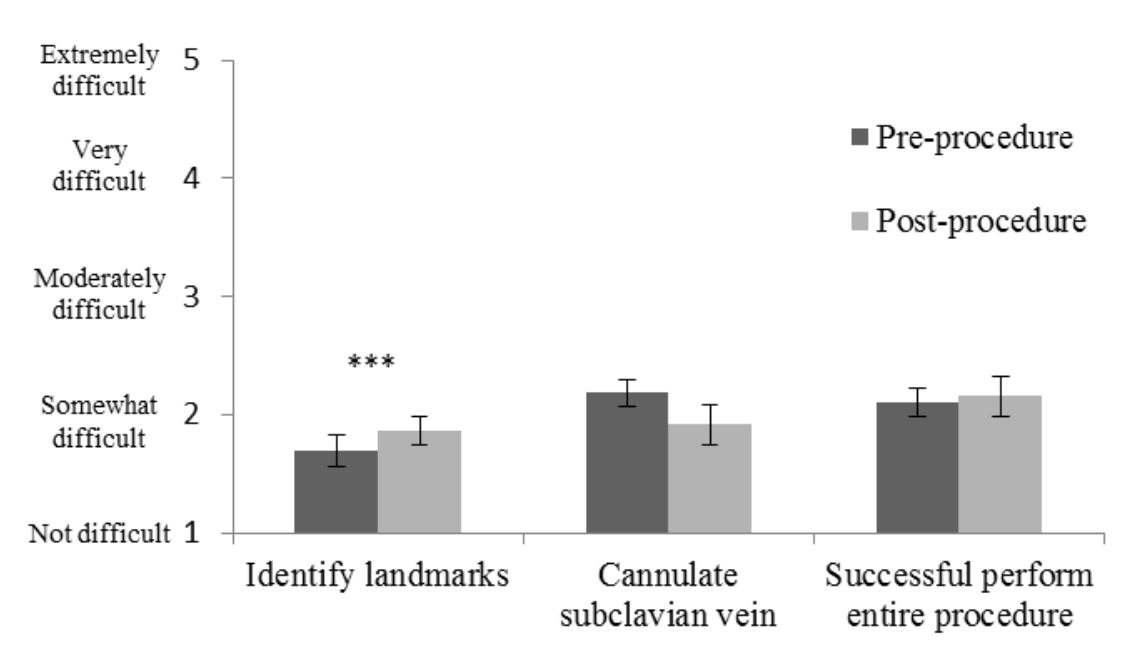

d. LVH repair

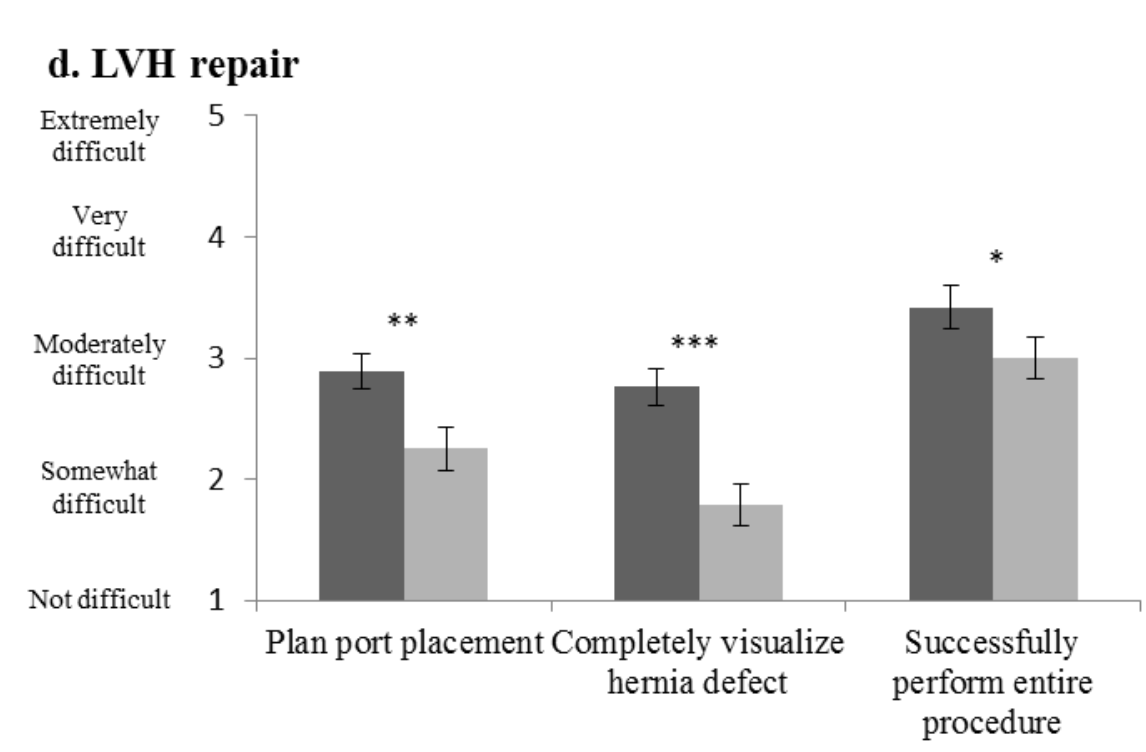

$$
\begin{aligned}
& * \mathrm{p}<.05 \\
& * * \mathrm{p}<.01 \\
& * * * \mathrm{p}<.001
\end{aligned}
$$

